\title{
The IOTA Expedition Results from the Total-Annular Eclipse in Gabon, 1987
}

\author{
Paul D. Maley
}

15807 Brookvilla, Houston, TX 77059, U.S.A.

\begin{abstract}
The results of the IOTA expedition to Gabon to observe the rare total-annular eclipse of 1987 March 29 were described. Multiple stations were used to determine data from Baily's Beads about the contact times. The techniques used and their application to future eclipses were evaluated. An additional aim was to develop a database to resolve corrections to the solar radius at specific epochs in order to determine the amplitude of time-dependent oscillations of the Sun's surface.
\end{abstract}

[The text was not received. - Eds.] 